\title{
High performance computing tools in science and engineering
}

\author{
Francisco Almeida · Jesús Vigo-Aguiar
}

Published online: 18 July 2013

(C) Springer Science+Business Media New York 2013

The combination of multicore architectures with the potential acceleration of Graphics Processing Units allows to design, low cost, desktop machines with hundreds of processing cores and at the same time to build large supercomputers with hundreds of thousands of computational units. Heterogeneity in the architecture and programming model appears naturally, so that the solution of many problems in science and engineering poses a different performance depending on the architecture and how they were programmed. Since current compilers are no longer able of automatically moving the computing power of the new generation of processors to applications, an interdisciplinary effort among researchers in science and engineering, and computing disciplines is demanded.

The purpose of the Minisymposium on High Performance Computing in Science and Engineering is to bring together applied mathematicians, computer scientists, and in general researchers with costly applications or a fair knowledge/interest in high performance computing, to present, share, and discuss their techniques, tools, and ideas in the area of high performance computing applied to complex large-scale computational problems. This Minisymposium use is to be held as part of the International Conference on Computational and Mathematical Methods in Science and Engineering (CMMSE).

This special issue collects a selection of the papers presented at the edition celebrated in La Manga del Mar Menor (Murcia-Spain) in July 2012 during the CMMSE-2012. Although several groupings of the contributions appearing in this issue can be performed, we decided to classify them into the four following groups.

F. Almeida

Dpt. Statistics and Computer Science, La Laguna University, Canary Islands, Spain

J. Vigo-Aguiar (凶)

Facultad de Ciencias, Univesisty of Salamanca, Salamanca, Spain

e-mail: jvigo@usal.es 
Solutions to a set of scientific applications demanding high performance computing are considered as part of this issue; they include the simulation of Brownian dynamics, pollutant transport in a shallow waters, and the improvement on multiple alignment of biological sequences.

The programmability wall problem facing researchers in the new scenario of these emergent architectures is also broached by researchers in this issue, automatic code generation of code based in the OpenACC standard, and the use of skeletal programming in specific domains are contributions attempting to bring architectures closer to the end user.

Parallel numerical algorithms for Multicore, Manycores, and GPUs have been also studied in this issue. The acceleration of the Lyapack library on GPUs and solutions to Block-Toeplitz linear systems of equations on multicores constitute improvements in this research area.

The importance of the architecture in high performance computing is highly analyzed in this issue. The analysis of the communication layer incorporates several research works in different scopes: security, where the secure multicast and the authentication problem in peer-to-peer platforms are revisited problems, signal processing, with the proposal of a tree-search detector for MIMO communications, and performance analysis, with the enhancement of network interfaces for web applications. Analyzing the performance of the processing units also delivers a set of papers dealing with modeling, performance analysis, and prediction. Load balancing in heterogeneous clusters and tuning applications into GPUs according to the block geometry are examples that illustrate these topics. Finally, modeling the energy consumption of parallel applications and the performance analysis of mobile devices explore two new promising research lines that are highly demanded under the new emergent platforms.

This special issue was made possible thanks to the participants in the Minisymposium and also to a significant number of reviewers that contributed the final result with their comments and suggestions for improvements. We would like to express our gratitude to all of them and we hope that the bottom line will be of your interest. We would also like to acknowledge to the Spanish Office of Science, for its support to the Spanish CAPAP-H3 network (HPC in Heterogeneous Systems, TIN2010-12011-E), and to the grant TIN2011-24598. 